\title{
Verwundbarkeit der offenen Gesellschaft
}

\author{
Carl Schmitt und Jacques Derrida in Zeiten des Terrors
}

Christian Bauer

Offene Gesellschaften sind etwas höchst Verwundbares. Ihre Offenheit macht sie prinzipiell verletzlich - und genau das ist der Preis ihrer Freiheit. Damit ist ein zentrales, existenziell herausforderndes Problem des politischen Diskurses der Gegenwart markiert: Terror als überall und jederzeit möglicher Ausnahmezustand, in welchem der Gegner als grundsätzlich tötbarer Feind erscheint. In komplementärer Differenz weisen Denker wie Carl Schmitt und Jacques Derrida verschiedene Wege aus dieser prekären Lage: ’homophiler Dissoziationen der Feindschaft unter Gleichen einerseits und 'heterophile< Assoziationen der Freundschaft unter Verschiedenen andererseits. Der transdisziplinäre Vulnerabilitätsdiskurs ${ }^{1}$ wird im Folgenden daher zunächst im politischen Feld der Gegenwart verortet, sodann mit entsprechenden Reflexionen von Schmitt sowie Derrida konstelliert und abschließend noch einmal auf das genannte Praxisfeld hin geöffnet. Sichtbar wird die potenzielle Verletzbarkeit offener Gesellschaften dabei nicht nur angesichts ihrer offensichtlichen Gegner, sondern auch ihrer vermeintlichen Freunde, die im Namen der Sicherheit soziale Freiheiten zu opfern bereit sind. Die entsprechenden Schlusssätze von Karl Poppers Die Feinde der offenen Gesellschaft ${ }^{2}$ sind bleibend aktuell:

"Wir können wieder zu Bestien werden. Aber wenn wir Menschen bleiben wollen, dann gibt es nur den [...] Weg in die offene Gesellschaft. Wir müssen ins Unbekannte, ins Ungewisse, ins Unsichere weiterschreiten und die Vernunft, die uns gegeben ist, verwenden, um, so gut wir es eben können, für beides zu planen: nicht nur für Sicherheit, sondern zugleich auch für Freiheit. $\ll^{3}$

\footnotetext{
1 Siehe einführend www.verwundbarkeiten.de (15.01.2017).

2 Vgl. Christian Bauer, Feinde der offenen Gesellschaft? Rechtspopulismus als theologisches Problem, in: www.feinschwarz.net (30. April 2016).

3 Karl Popper, Die offene Gesellschaft und ihre Feinde, Bd. 1: Der Zauber Platons, Tübingen ${ }^{7} 1992,239$.
} 


\section{Terror - eine Bedrohung der offenen Gesell- schaft}

"Du wirst mich nicht töten« - dieses zwischenmenschliche Grundvertrauen wird gerade gewaltig erschüttert. Denn es ist inzwischen ja alles andere als sicher, dass der oder die Andere dort neben mir mich nicht töten wird. Entsprechender Terror beginnt im Kopf. Zum Beispiel dann, wenn man sich überlegt, den Thalys nach Paris zu nehmen. Oder ob man zum public viewing geht. Ich kenne noch jenes mulmige Gefühl, das mich stets erfasst hat, wenn ich unmittelbar nach dem 11. September 2001 an die Berliner TU geradelt bin - vorbei an der massiv bewachten US-Botschaft. Dass seit dem Sommer 2016 nun auch ausgerechnet meine idyllische Heimatstadt Würzburg in einem Atemzug mit bekannten europäischen Terrorzielen wie Istanbul, Paris, Brüssel oder Nizza genannt wird, macht die ganze Sache nicht besser. Sascha Lobo bemerkte kurz danach, eine entsprechende "Überprüfung der potenziellen Selbstbetroffenheit ${ }^{4}$ sei ein lebensweltlich nachvollziehbares "Reaktionsmuster auf den Axt-Angriff bei Würzburg, dessen neue Qualität die Nähe ist ${ }^{5}$. Die alläglichen Konsequenzen des Terrors berühren längst auch die eigene Lebenswelt:

"Der Terrorismus dieser Zeit wird weit nach vorne wirken, ins Leben jedes Einzelnen, er verlangt eine Haltung. [...] Wie mutig muss man sein, um frei zu bleiben? Wie vorsichtig, um sicher zu sein? Kann man es überhaupt Freiheit nennen, wenn dazu das Risiko gehört, von dritter Hand zu sterben, als Opfer der Terroristen? $\ll^{6}$

In dieser Situation wird ein brillanter, zugleich aber auch höchst gefährlicher politischer Denker wieder aktuell - ein >Meister aus Deutschland , der einst als Hitlers Kronjurist galt: Carl Schmitt (1888-1985). Im Anschluss an ihn lässt sich sagen: Terror ist ein jederzeit möglicher Ausnahmezustand, dessen gewaltsame »Entfesselung der reinen Feindschaft « ${ }^{7}$ keine Menschen mit unantastbarer

\footnotetext{
4 Sascha Lobo, Wir sind Terroregozentriker http://www.spiegel.de/netzwelt/netzpolitik/attentate-wir-sind-alle-terroregozentriker-a-1103888.html (29. Januar 2017).

5 Ebd.

${ }^{6}$ Ralf Wiegand, Klick. Die Welt hat den Terror jetzt verstanden, in: Süddeutsche Zeitung, 16./17. Januar 2016, 3.

7 Jacques Derrida, Politik der Freundschaft, Frankfurt a.M. 2002, 184 (im Folgenden PdF).
} 
Würde kennt, sondern nur unlawful combatants ${ }^{8}$, die man wie die antike Rechtsfigur des homo sacer $^{9}$ konsequenzfrei verletzen oder gar töten kann. Offene Gesellschaften müssen auf die entsprechende Herausforderung ihrer vergleichsweise freien Lebensform souverän reagieren. Diese politische Souveränität darf ihnen nicht entgleiten, denn Schmitt zufolge gilt ja richtigerweise: »Souverän ist, wer über den Ausnahmezustand bestimmt. $\|^{10}$ Dass man Terroristen keine Macht über das eigene Leben einräumen darf, hat handfeste psychologische Gründe:

»Wer sich der Angst hingibt, der meidet am Anfang vielleicht nur Kaufhäuser, dann größere Menschenansammlungen, danach kleinere. Irgendwann sind ihm dann schon drei Leute zuviel, und am Ende fährt er jeden Tag direkt von der Arbeit nach Hause und weiß gar nicht mehr, was das Leben ausmacht. ${ }^{11}$

Die beste Reaktion aufTerroranschläge ist daher eine postheroische Indifferenz, wie sie Herfried Münkler nahelegt:

"Die besteht darin, dass wir schnell vergessen. Unsere mediale Welt und ihre Unterhaltungsangebote [...] führen dazu, dass solche Anschläge, und mögen sie noch so furchtbar sein, sich nach einiger Zeit auch wieder verlieren. Und wenn eine Gesellschaft in der Lage ist, eine Weile nach einem solchen Anschlag so zu tun, als habe es ihn gar nicht gegeben, das heißt ihr Leben wieder so aufzunehmen und fortzuführen, wie sie das vorher geplant hat, dann ist das eine Erfahrung des Widerstandleistens [...], die ausgesprochen effektiv ist. Weil sich dabei herausstellt, wie schwach doch letzten Endes die angreifenden Akteure sind. Sie sind stark nur in dem Augenblick, in dem unsere [...] Nervosität, ja womöglich unsere Hysterie wie Schlagkraftverstärker wirken. $\aleph^{12}$

Angesichts seiner potenziellen Gefährdung ist am freiheitlichen Lebensstil einer zwar terroristisch verwundbaren, im Sinn Münk-

\footnotetext{
8 Vgl. Carl Schmitt, Theorie des Partisanen, Berlin ${ }^{52002,77 .}$

9 Vgl. Giorgio Agamben, Homo sacer. Die souveräne Macht und das nackte Leben, Frankfurt a.M. 2001.

${ }^{10}$ Carl Schmitt, Politische Theologie, Vier Kapitel zur Lehre von der Souveränität, Berlin ${ }^{8} 2004,13$.

${ }^{11}$ Christina Berndt, Entspannt euch. Seit den Gewalttaten von Würzburg, München und Ansbach greift die Angst wie ein Virus um sich, in: Süddeutsche Zeitung, 30./31. Juli 2016, 3 .

${ }^{12}$ Herfried Münkler, Im Zwischenzustand, in: http://www.sueddeutsche.de/kultur/ herfried-muenkler-im-zwischenzustand-1.2739211?reduced=true (29. Januar 2017).
} 
lers aber eben auch starken offenen Gesellschaft festzuhalten: "Eine Gesellschaft, die sich nicht einschüchtern lässt, die Opfer aushält und ihr offenes Leben weiterlebt, ist vom Terror nicht zu besiegen. ${ }^{13}$ Karl Popper zitiert in seinem Buch Die Feinde der offenen Gesellschaft (1945) überraschend aktuelle Worte aus einer bei Thukydides überlieferten Rede des Perikles:

"Unsere Stadt steht der Welt offen; wir vertreiben nie einen Fremdling. [...] Wir sind frei, genauso zu leben, wie es uns gefällt, und doch sind wir immer bereit, jeglicher Gefahr ins Auge zu sehen. [...] Wir lieben die Schönheit, ohne uns Träumen hinzugeben, und obgleich wir versuchen, unseren Verstand zu stärken, so schwächen wir doch nicht dadurch unseren Willen. [...] Wir halten das Glück für die Frucht der Freiheit und die Freiheit für die Frucht der Tapferkeit, und wir schrecken nicht vor der Gefahr des Krieges zurück. .14 $^{14}$

Nach den Anschlägen von Paris im November 2015, in deren Kontext immer wieder gefordert wurde, Europa müsse nun seine façon de vivre verteidigen, bewiesen diese Worte eine erstaunliche gegenwartspolitische Aktualität:

"Ein Terroranschlag in Paris hat eine symbolische Bedeutung, die jeden Bürger der westlichen Welt ins Herz treffen muss. Es geht, wie sofort und zu Recht gesagt wurde, um sunsere Art zu leben<. Es geht um ein welthistorisch ziemlich spätes, ziemlich einzigartiges Amalgam von Freiheit und Lebensfreude, Aufklärung und Hedonismus $[\ldots] . \ll^{15}$

Dem entgegengesetzter Terror betrifft, wie kurz darauf die Anschläge von Istanbul im Januar 2016 zeigten, nicht nur den Westen Europas:

"Paris und Istanbul mögen ein paar tausend Kilometer auseinanderliegen, aber sie sind doch Symbole einer gemeinsamen Sache. Diese freien Städte voller Lust und Leben, sie sind jetzt verwundete Körper. Sie sind Leidensgenossen. $\aleph^{16}$

\footnotetext{
${ }^{13}$ Stefan Ulrich, Kriegszone Europa, in: Süddeutsche Zeitung, 5. April 2004, 4.

${ }_{14}$ Zit. nach Popper, Die offene Gesellschaft und ihre Feinde, 222.

${ }_{15}$ Gustav Seibt, Reaktion auf den Terror. Der nächste Glaubenskrieg, in: http://www. sueddeutsche.de/kultur/debatte-um-werte-unsere-art-zu-leben-1.2739209 (29. Januar 2017).

${ }^{16}$ Ralf Wiegand, Klick. Die Welt hat den Terror jetzt verstanden, in: Süddeutsche Zeitung, 16./17. Januar 2016, 3.
} 
Das wohl souveränste Zeichen einer zwar verwundbaren, aber dennoch starken »Macht in der Ohnmacht ${ }^{17}$ stammt von Antoine Leiris, einem französischen Journalisten, dessen Frau Hélène im November 2015 ermordet worden war:

"Freitag Abend habt ihr das Leben eines außerordentlichen Wesens geraubt, das der Liebe meines Lebens, der Mutter meines Sohnes, aber meinen Hass bekommt ihr nicht. [...] Zugegeben, der Kummer zerreisst mich, diesen kleinen Sieg habt ihr errungen, aber er wird von kurzer Dauer sein. Ich weiß, dass [...] wir uns in jenem Paradies der freien Seelen wiederbegegnen werden, zu dem ihr niemals Zutritt haben werdet. Wir sind zwei, mein Sohn und ich, aber wir sind stärker als alle Armeen der Welt. Ich will euch jetzt keine Zeit mehr opfern, ich muss mich um Melvil kümmern, der gerade aus seinem Mittagsschlaf aufgewacht ist. Er ist gerade mal siebzehn Monate alt; er wird seinen Nachmittagssnack essen wie jeden Tag, dann werden wir wie jeden Tag zusammen spielen, und sein ganzes Leben lang wird dieser kleine Junge euch beleidigen, weil er glücklich ist und frei. Denn, nein, auch seinen Hass bekommt ihr nicht. ${ }^{18}$

\section{Carl Schmitt - >homophile (Dissoziationen der Feindschaft}

Carl Schmitt war ein deutscher Partisan des Weltgeistes, dessen zum Teil analytisch herausragende, in seinen Konsequenzen jedoch brandgefährliche Theorie souveräner Entscheidung über den Ausnahmezustand entlang klarer Unterscheidungen von Freundschaft und Feindschaft im gegenwärtigen Terror, aber auch in der politischen Reaktion darauf zunehmend an Bedeutung gewinnt. So stellt auch die Politik der gegenwärtigen US-Administration eine massive Bewährungsprobe für das Konzept einer universalen Kommunikationsgemeinschaft im herrschaftsfreien Diskurs dar - Donald Trump markiert die dezisionistische Grenze aller bürgerlich-liberalen Diskursivität à la Jürgen Habermas. Er simuliert die scheinbar wiedergewonnene Handlungsmacht politischer Führung ("take back control«)

${ }^{17}$ Vgl. Hans-Joachim Sander, Macht in der Ohnmacht. Eine Theologie der Menschenrechte, Freiburg i.Br. 1999.

${ }_{18}$ Antoine Leiris, Meinen Hass bekommt ihr nicht, München 2016, 59-61. Allein die hier angedeutete Eschatologie einer ausgleichenden Gerechtigkeit für Täter und Opfer wäre es wert, wissenschaftstheologisch ausgewertet und weiterverfolgt zu werden. 
mittels einer "Erfindung des Feindes" (PdF, 125), die unsere globale Ordnung der Dinge ganz im Sinne Schmitts zu "re-politisieren (PdF, ebd.) versucht. Dessen zentrales politiktheoretisches "Axiom" (PdF, ebd.) besteht in jener »Unterscheidung von Freund und Feind « ${ }^{19}$ in Krieg und Frieden, durch die er das Politische als solches definiert:

"Wo Krieg und Feindschaft sicher bestimmbare [...] Erscheinungen sind, kann alles, was nicht Krieg ist [...]: Friede, was nicht Freund ist [...]: Feind heißen. Umgekehrt:Wo Friede und Freundschaft [...] das Gegebene sind, kann alles, was nicht Friede ist: Krieg, und was nicht Freundschaft ist: Feindschaft werden. Im ersten Fall ist der Friede, im zweiten Fall der Krieg von dem bestimmt Gegebenen her negativ bestimmt. Im ersten Fall ist aus demselben Grunde Freund der Nicht-Feind, im zweiten Falle Feind der Nicht-Freund. $\|^{20}$

Das Politische stellt für Schmitt "kein eigenes Sachgebiet, sondern nur den Intensitätsgrad von Assoziation oder Dissoziation ${ }^{21}$ dar, wobei in dessen alles einschließender Totalität Freundschaft und Feindschaft den jeweils "äußersten Intensitätsgrad $\aleph^{22}$ von Assoziation oder Dissoziation markieren. Beide repräsentieren eine Intensität, in der buchstäblich alles politisch werden kann, sobald es in den Horizont des Ausnahmezustands gerät: "Das Politische ist das Totale. $«{ }^{23}$ Schmitt bestimmt es von jenem Ernstfall her, welcher den Krieg als eine Schlüsselmetapher für das Politische im Extrem seiner möglichen Intensitäten erweist: »Politik ist die Fortsetzung des Krieges mit anderen Mitteln ${ }^{24}$. Diese Umkehrung des geflügelten Wortes von Clausewitz durch Michel Foucault offenbart die Permanenz jenes ewigen Widerstreits, dessen fundamentale Differenzen keine schlichtende Metainstanz kennen ${ }^{25}$ - eine prinzipiell richtige Auffassung, die bei Schmitt ihr wohl denkbar gefährlichstes Extrem findet: Der Krieg der absoluten Feindschaft kennt keine Hegung. ${ }^{26}$

\footnotetext{
${ }^{19}$ Schmitt, Der Begriff des Politischen, 26.

20 Ebd., 105.

${ }^{21}$ Ebd., 38.

22 Ebd., 27.

${ }^{23}$ Vgl. Schmitt, Politische Theologie, 7.

${ }^{24}$ Michel Foucault, In Verteidigung der Gesellschaft,Vorlesung am Collège de France 1975-1976, Frankfurt a.M. 1999, 26.

${ }^{25}$ Vgl. Christian Bauer, Differenzen der Spätmoderne? Theologie vor der Herausforderung der Gegenwart, in: Stefan Gärtner/Tobias Kläden/Bernhard Spielberg (Hg.), Praktische Theologie in der Spätmoderne. Herausforderungen und Entdeckungen, Regensburg 2013, 27-47.

${ }^{26}$ Schmitt, Theorie des Partisanen, 56.
} 
Der Jurist benennt aber auch die mögliche Gegenposition zu diesem Ernstfall des Politischen:

»Die Hegung und klare Begrenzung des Krieges enthält eine Relativierung der Feindschaft. Jede solche Relativierung ist ein großer Fortschritt im Sinne der Menschheit. « ${ }^{27}$

Die politische Ökonomie von Freundschaft und Feindschaft ist bei Schmitt prinzipiell asymmetrisch strukturiert. Sie bilden eine politische Grunddifferenz, die er selbst nur dissoziierend von der Feindschaft her, nicht aber assoziierend von der Freundschaft her zu denken vermag. Freundschaft ist für ihn primär nicht eigenständig definierbare Nicht-Feindschaft - und die "feindliche Frage" (PdF, 206) danach ist Derrida zufolge eine "verletzende und verletzte Frage, eine Verletzungsfrage« (PdF, 211):

"Ich stelle sie mir erst, wenn sie auf mich einstürzt, im Angriff und in der Kränkung. Im Verbrechen oder in der Beschädigung oder im Vorwurf. Die Frage verletzt mich, sie ist eine in mir selbst aufbrechende Wunde.« (PdF, 206)

\section{Jacques Derrida - >heterophile، Assoziationen der Freundschaft}

Jacques Derrida denkt die Freund-Feind-Differenz ebenso asymmetrisch wie Carl Schmitt - nur eben assoziierend von der Freundschaft her. Dabei hat er eine Etymologie auf seiner Seite, auf die auch Schmitt verweist: in romanischen Sprachen wird der Feind "nur negativ bestimmt als Nicht-Freund ${ }^{28}$ - so zum Beispiel im Lateinischen (»amicus-inimicus ${ }^{29}$ ), im Italienischen (»amico-nemico ${ }^{30}$ ) oder im Französischen (»ami-ennemi $\left.{ }^{31}\right)$. Mit seinem Buch Politiques de l'amitié (1994) dekonstruiert Derrida in entsprechender Weise Schmitts bereits zitiertes Werk Der Begriff des Politischen (1932), wobei das politische Zeugnis der Freundschaft ("philia") für ihn weniger eine Angelegenheit unter Gleichen ("Homophilie») als vielmehr unter Ungleichen ("Heterophilie») ist. Schmitt hingegen stelle das Politische prinzipiell unter ein dissoziierendes "Gesetz der

\footnotetext{
${ }^{27}$ Schmitt, Der Begriff des Politischen, 11.

${ }^{28}$ Ebd., 105.

${ }^{29}$ Ebd.

${ }^{30}$ Ebd.

${ }^{31}$ Ebd.
} 
Homophilie und des Autochthonen" (PdF, 154), dessen Exklusionen zugleich auf »staatsbürgerliche Gleichheit (Isonomie)« (PdF, ebd.) und "Gleichheit der Geburt (Isogonie)« (PdF, ebd.) gegründet seien. Karl Popper identifizierte dieses Denken einer Tribalisierung im Sinne einer politisch dissoziiierenden My-tribe-Ideologie als einen wesentlichen Gegensatz zur offenen Gesellschaft:

"Eine geschlossene Gesellschaftsordnung ähnelt immer einer Herde oder einem Stamm; sie ist eine halborganische Einheit, deren Mitglieder durch halbbiologische Bande [...] zusammengehalten werden $[\ldots],[\ldots]$ nicht bloß durch abstrakte soziale Beziehungen wie Arbeitsteilung, Gütertausch, sondern durch konkrete physische Beziehungen [...]..132

Derrida möchte in seiner doppelten "Entgegnung auf das Projekt Carl Schmitts" (PdF, ebd.) die Demokratie genau dieser "autochtonen und homophilen Verwurzelung" (PdF, ebd.) entreißen. Zum einen im Sinne einer "notwendigen Entpolitisierung" (PdF, 155), die sich in keinen absoluten Gegensatz von Freundschaft und Feindschaft drängen lässt. Und zum anderen im Sinne eines "Versuchs, durch diese Entpolitisierung (PdF, ebd.) eine »andere Politik, eine andere Demokratie zu denken" (PdF, ebd.) - eine "kommende und im Kommen bleibende Demokratie» (PdF, 154), welche die sozialen Bande von Klassenzugehörigkeit, Herkunft und Geschlechtlichkeit sprengt. Schon die athenische Demokratie hatte sich durch den Ausschluss von Sklaven, Frauen und Fremden homogenisiert. Sie gilt es im Sinne einer universalen "Idee der Gerechtigkeit ${ }^{33}$ auf eine kommende Demokratie hin zu öffnen, welche die »Freundschaft aus und von einer das Recht transzendierenden Gerechtigkeit" (PdF, 249) in politische Prozesse überführt: "Die Gerechtigkeit bleibt im Kommen, sie muss noch kommen, sie hat, sie ist Zukunft, sie ist die Dimension ausstehender Ereignisse, deren Kommen irreduktibel ist. $\mathbb{1}^{34}$ Sein eigenes philosophisches Projekt einer Dekonstruktion aller Dinge bis an den Ursprung ihrer anfänglichen différance ("keine Dekonstruktion ohne Demokratie und keine Demokratie ohne Dekonstruktion«) (PdF, 156) weiß Derrida mit diesen politischen Optionen verknüpft:

"Sie würde sich auf das Vertrauen, den Kredit, den Glauben [...] richten, die man der Abstammung, der Geburt und der Herkunft

${ }^{32}$ Popper, Die offene Gesellschaft und ihre Feinde, 207.

${ }^{33}$ Jacques Derrida, Gesetzeskraft. Der 'mystische Grund der Autorität‘, Frankfurt a.M. 1991, 52.

${ }^{34}$ Derrida, Gesetzeskraft, 52. 
[...] zuteil werden lässt [...]. Nicht, um Krieg gegen sie zu führen oder in ihnen das schlechthin Böse auszumachen, sondern um eine Politik, eine Freundschaft, eine Gerechtigkeit zu leben, an deren Anfang der Bruch mit ihrer naturhaften Homogenität [...] steht. Die also dort anfangen, wo der Anfang eine Spaltung und einen Aufschub [...] zeitigt. Die damit anfangen, eine sursprünglicheく Heterogenität zu markieren [...], um sie zu öffnen, und sei es auch für sich selbst zu öffnen."(PdF, 156)

Diese »dekonstruktive Auto-Delimitation« (PdF, ebd.) der Demokratie, bei der nicht Feindschaft, sondern Freundschaft am "Anfang der Politik« (PdF, 249) steht, zeigt, wie sehr in Zeiten des Terrors die Sache des Denkens selbst auf dem Spiel steht:

"Die Freundschaft als Philosophie, die Philosophie als Freundschaft, die philosophische Freundschaft, die Freundschafts-Philosophie - das wird im Abendland stets ein in sich unauflöslicher Begriff gewesen sein. Keine Freundschaft ohne eine Art philosophia, keine Philosophie ohne philia. [...] Jetzt dagegen fordert Schmitt uns auf, den Krieg, also die Tötung und letztlich das, was er die absolute Feindschaft nennt, als Sache der Philosophie zu denken $[\ldots] . \ll(\mathrm{PdF}, 200)$

\section{Politik der Freundschaft - Differenzen jenseits des Gegensatzes}

Angesichts der terroristischen Verletzbarkeit einer offenen Gesellschaft kann man also prinzipiell mindestens zwei Wege beschreiten: man kann entweder >den Schmitt machen (Diskurse homophil abgrenzen) oder aber >den Derrida`(Diskurse heterophil offenhalten). Wenn man das Politische mit Schmitt "von der Feindschaft" (PdF, 210) her bestimmt, so geht man dabei "vom Gegensätzlichen selbst« (PdF, ebd.) aus. Freundschaft und Feindschaft sind, mit Gilles Deleuze gesprochen, ${ }^{35}$ aber eine Differenz im Sinne Jacques Derridas und kein Gegensatz im Sinne Carl Schmitts - und auch diese Entgegensetzung von Gegensatz und Differenz selbst ist eine Differenz und kein Gegensatz:

${ }^{35}$ Vgl. Gilles Deleuze, Différence et répétition, Paris ${ }^{12} 2011$, 1 sowie Vincent Descombes, Das Selbe und das Andere. Fünfundvierzig Jahre Philosophie in Frankreich (1933-1978), Frankfurt a.M. 1981, 193; 195. 
»Die Differenz, die wir suchen, ist die zwischen der Differenz und dem Gegensatz. [...] Vom Gesichtspunkt der Bejahung ist dieVerneinung nicht das Gegenteil der Bejahung. Das Nein setzt sich dem Ja nicht entgegen, sondern zeigt eine Differenz an. ${ }^{36}$

Es gilt, die »Gegensatzlogik» (PdF, 332f.) Schmitts mit Derrida in Richtung der Differenz einer Bejahung offener Freundschaft zu überschreiten:

"Entgegensetzen kann man dieser Logik [...] nichts anderes als den Gegensatz [oder besser: ihre Differenz]: Was für den Feind zutrifft (ich kann oder muss dich töten, und umgekehrt), wird von der Freundschaft [...] außer Kraft gesetzt [...]. Es kann also keineswegs schon entsprechend vom Freund gelten, was vom Feind gilt $[\ldots] . \lll(\mathrm{PdF}, 173)$

Wer den gewaltbesetzten Freund-Feind-Gegensatz Schmitts wie Derrida von der Freundschaft her öffnet, muss den Exklusionen jener "absoluten Feindschaft" (PdF, 189) standhalten, welche die Gegner einer offenen Gesellschaft gegenwärtig radikalisiert und zu ihren erbitterten Feinden macht. Denn die Freunde einer homophil geschlossenen Gesellschaft sind die Feinde einer ’heterophik offenen. Die Freunde einer offenen Gesellschaft jedoch sind nicht die Feinde einer geschlossenen - sie sind nur deren Gegner. Es geht um einen differenzfähigen, alteritätsoffenen Widerstreit jenseits geschlossener Verbrüderung von Gleichen, bei dem aus "feindlichen Brüdern« (PdF, 206) freundliche Gegner werden. Wer im Sinne Derridas für entsprechende politische Optionen eintritt, macht sich prinzipiell verwundbar. In diesen Zusammenhang wird eine weitere Unterscheidung relevant. Für den Begriff des Opfers gibt es in vielen Sprachen nämlich zwei Worte - im Englischen zum Beispiel victim und sacrifice ${ }^{37}$. Und sie machen einen Unterschied. Feindschaft nämlich ist mit der Bereitschaft verbunden, den anderen zum Opfer im Sinne von victim zu machen. Freundschaft hingegen mit der Bereitschaft, sich selbst anstelle des anderen im Sinne von sacrifice zu opfern. Freundschaft umfasst daher die Bereitschaft, eher sich selbst verwunden zu lassen ("sacrifice") als andere Menschen $\mathrm{zu}$ verwunden ("victimisation«). Feindschaft jedoch die Bereitschaft, eher andere $\mathrm{zu}$ verwunden als sich selbst verwunden zu lassen - so wie auch Jacques Derrida mit Carl Schmitt gegen ihn schreibt: „Kei-

\footnotetext{
${ }^{36}$ Descombes, Das Selbe und das Andere, 193f.

${ }^{37}$ Vgl. Hans-Joachim Sander, Nicht verleugnen. Die befremdende Ohnmacht Jesu, Würzburg 2001, 47f.
} 
ne Freundschaft ohne mögliche Verletzung." (PdF, 211) Diese unterschiedliche Intensität von aktiver und passiver Verwundungsbereitschaft verweist auf eine großartige Metapher, die Georges Bataille für die Freundschaft gefunden hat. Er fasst sie als einen Riss »in der Rüstung ${ }^{38}$ zwischenmenschlicher Nahbeziehungen: „Sie erfordert eine Koinzidenz von zwei Rissen, in mir selbst und im anderen. ${ }^{39}$

\section{Christliche Theologie - eine Ressource gesell- schaftlicher Offenheit}

Die wehrhafte Demokratie einer offenen Gesellschaft verteidigt ihren entsprechenden Lebensstil gegen alle Feinde, die sie als handlungsunfähige "Quatschbude» diffamieren: "Wir halten Diskussionen nicht für einen Stein des Anstoßes auf dem Wege der politischen Aktion, sondern für eine unentbehrliche Vorbereitung zum weisen Handeln. $\aleph^{40}$ Dieses Handeln, von dem Karl Popper noch mit ungebrochenem Pathos sprach, ist in spätmodernen Zeiten nicht mehr unterhalb eines gewissen Komplexitätsgrades zu haben. Denn die größten Feinde einer offenen Gesellschaft sind heute nicht nur islamistische Religionsfanatiker, sondern in komplementär feindlicher Entgegensetzung auch populistische Rechtspolitiker. Beide stehen für große Erzählungen, welche die Komplexität unserer Gesellschaft mit ihren abertausend kleinen Geschichten des Lebens auf ein einfaches, orientierendes Narrativ zu reduzieren versuchen: les extrèmes se touchent. Auch in dieser Hinsicht gilt: "Der Feind ist unsere eigene Frage als Gestalt. ${ }^{41}$ Die Antwort auf die komplexen Fragen unserer von Terror geprägten Gegenwart jedenfalls fällt in beiden Fällen ähnlich einfach und in ihrer Einfachheit leider auch erschreckend falsch aus. Die aktuellen Fluchtbewegungen verbinden beide Extreme auf eine fast schon tragische Weise, an der nicht zuletzt auch das bürgerliche Gesicht rechtspopulistischer Brandstifter erschreckt: Biedermänner und Biederfrauen, die im Angesicht des Terrors Flüchtlinge und nicht Fluchtursachen bekämpfen. Religionsfanatiker und Rechtspopulisten verkörpern in ihrer jeweiligen Gestalt aber nicht nur die Frage des jeweils anderen, sie berühren in brutaler Radikalität auch die offenen Grundfragen westlicher Ge-

${ }^{38}$ Georges Bataille, Die Freundschaft und Das Halleluja. Atheologische Summe II, München 2002, 43.

${ }^{39}$ Bataille, Die Freundschaft und Das Halleluja, 43.

${ }^{40}$ Perikles (zit. nach Popper, Die offene Gesellschaft und ihre Feinde, 222).

${ }^{41}$ Schmitt, Theorie des Partisanen, 87. 
sellschaften:Wovon leben wir eigentlich und wofür? Wie gelingt ein gutes Leben für möglichst viele Menschen? Und wer ist eigentlich dieses $>$ wir ${ }^{42}$ auf seiner Suche nach dem kleinen Glück in dieser Zeit? Bei der Beantwortung dieser existenziellen Fragen gilt es, an den Errungenschaften einer offenen Gesellschaft festzuhalten und sie um den Preis eigener Verwundbarkeit gegen ihre Feinde und deren Gewalt offenzuhalten: »Haben wir auf dem gleichen vitalen Niveau der Hingabe etwas anderes entgegenzusetzen? « $^{43}$

Dabei könnte man durchaus auch auf gesellschaftlich verschüttete theologische Ressourcen des Christentums zurückgreifen, das sich selbst ein »offenes Narrativ « ${ }^{44}$ menschlicher und göttlicher Vulnerabiltät verstehen lässt. Und zwar beginnend mit der inkarnatorischen Verletzbarkeit Gottes ${ }^{45}$ in der Gestalt Jesu, in dessen Nachfolge dann aber auch in der Ekklesiologie - beispielsweise in Gestalt einer grandiosen Formulierung Henri de Lubacs, der schon 1938 den Katholizismus als eine »Wirklichkeit ${ }^{46}$ sui generis bezeichnete, die, "um zu sein, es nicht nötig hat, sich entgegenzusetzen ${ }^{47}$ : Theologie und Kirche treten ins existenziell Offene ${ }^{48}$. Das Zweite Vatikanische Konzil vorwegnehmend, das entsprechend "positive, nichtexklusive Aussagen « ${ }^{49}$ zum Wesen des Katholischen lehrte, sprach Hans Urs von Balthasar bereits 1951 in Schleifung der Bastionen von einem verwundbar machenden, inkarnationstheologisch ${ }^{50}$ jedoch

\footnotetext{
${ }^{42}$ Vgl. Thomas Dienberg/Thomas Eggensperger/Ulrich Engel (Hg.), Auf der Suche nach einem neuen 'Wir . Theologische Beiträge zu Gemeinschaft und Individualisierung, Münster 2016.

${ }^{43}$ Ottmar Fuchs, Identität im Horizont christlichen Glaubens. Theologische Annäherungen, in: Hans Poser/Bruno Reuer (Hg.), Bildung - Identität - Religion. Fragen zum Wesen des Menschen, Berlin 2004, 99-118, 117.

${ }^{44}$ Lieven Boeve, Lyotard and theology, London/New York 2014, 89.

45 Antoine Leiris an die Mörder seiner Frau - und auch diese schöpfungstheologische Aussage gälte es, theologisch weiterführend zu würdigen: "Wenn der Gott, für den ihr blind tötet, uns nach seinem Ebenbild geschaffen hat, dann muss jede Kugel, die den Körper meiner Frau getroffen hat, eine Wunde in sein Herz gerissen haben." (Leiris, Meinen Hass bekommt ihr nicht, 59).

${ }^{46}$ Henri de Lubac, Glauben aus der Liebe, übertragen und eingeleitet von Hans Urs von Balthasar, Einsiedeln ${ }^{3} 1992,263$.

${ }^{47}$ Lubac, Glauben aus der Liebe, 263.

48 Vgl. Christian Bauer, Konstellative Pastoraltheologie. Erkundungen zwischen Diskursarchiven und Praxisfeldern, 424-428.

${ }^{49}$ Walter Simonis, Glaube und Dogma der Kirche. "Lobpreis seiner Herrlichkeit" (Eph 4,14). Leitfaden der katholischen Dogmatik nach dem Zweiten Vatikanum, St. Ottilien 1995, 8; 26.

${ }^{50}$ Im Motiv der Fleischwerdung Gottes ist eine prinzipielle Verletzbarkeit seines Wesens fast schon rautomatisch mitgegeben.
} 
indispensablen "Herabsteigen der Kirche in die Fühlung mit der Welt «" ${ }^{51}$ : Sinkende Mauern können vieles begraben, das durch sie geschützt zu leben schien; aber die Fühlung mit dem Raum, die sich herstellt, ist größer. «2 Eine erhöhte Verwundbarkeit ist der notwendige Preis dieser neuen pastoralen Weltfühligkeit einer Kirche, die nun im letzten keine Mauern mehr um sich ${ }^{53}$ hat und daher »wehrlos offen zur Welt «" ${ }^{54}$ steht. Für sie gilt jenes Prinzip der "Nicht-Ausschließung ${ }^{55}$, welches das Zweite Vatikanum am Ende der Pastoralkonstitution Gaudium et spes in Form eines feierlichen Versprechens formuliert und das verschiedene Spielräume eines in seiner verletzbaren Offenheit beispielhaft "aufrichtigen Dialogs" (GS 92) der Kirche mit der Welt ermöglicht:

»Der Wunsch nach einem solchen Dialog [...] schließt unsererseits niemanden aus, weder jene, die hohe Güter der Humanität pflegen [...], noch jene, die Gegner der Kirche sind und sie auf verschiedene Weise verfolgen." (GS 92)

- Christian Bauer ist Professor für Pastoraltheologie und Homiletik an der Katholischtheologischen Fakulät der Universität Innsbruck. Seine Forschungsinteressen bewegen sich im Feld einer spätmodern ausgerichteten Gegenwartstheologie der christlichen Zeitgenossenschaft.

\footnotetext{
${ }^{51}$ Hans Urs von Balthasar, Schleifung der Bastionen. Von der Kirche in dieser Zeit, Einsiedeln 1954, 83.

${ }^{52}$ Balthasar, Schleifung der Bastionen, 83.

${ }^{53}$ Hans Urs von Balthasar, Eucharistischer Kongress, in: ders., Sponsa verbi. Skizzen zur Theologie II, Einsiedeln 1961, 514-524, hier 518.

${ }^{54}$ Balthasar, Eucharistischer Kongress, 518.

${ }^{55}$ Hans-Joachim Sander, Von der kontextlosen Kirche im Singular zur pastoralen Weltkirche im Plural - ein Ortswechsel durch Nicht-Ausschließung prekärer Fragen, in: Peter Hünermann/Bernd-Jochen Hilberath (Hg.), Die Dokumente des Zweiten Vatikanischen Konzils. Theologische Zusammenschau und Perspektiven, Freiburg i.Br. 2005, 383-394, 390ff.
} 